\title{
STABILITY CRITERIA FOR FLUID FLOWS
}


Published*:

Vol. 68 Differential Equations, Bifurcations, and Chaos in Economics by W. B. Zhang

Vol. 69 Applied and Industrial Mathematics in Italy eds. M. Primicerio, R. Spigler and V. Valente

Vol. 70 Multigroup Equations for the Description of the Particle Transport in Semiconductors by M. Galler

Vol. 71 Dissipative Phase Transitions eds. P. Colli, N. Kenmochi and J. Sprekels

Vol. 72 Advanced Mathematical and Computational Tools in Metrology VII eds. P. Ciarlini et al.

Vol. 73 Introduction to Computational Neurobiology and Clustering by B. Tirozzi, D. Bianchi and E. Ferraro

Vol. 74 Wavelet and Wave Analysis as Applied to Materials with Micro or Nanostructure

by C. Cattani and J. Rushchitsky

Vol. 75 Applied and Industrial Mathematics in Italy II eds. V. Cutello et al.

Vol. 76 Geometric Control and Nonsmooth Analysis eds. F. Ancona et al.

Vol. 77 Continuum Thermodynamics by K. Wilmanski

Vol. 78 Advanced Mathematical and Computational Tools in Metrology and Testing eds. F. Pavese et al.

Vol. 79 From Genetics to Mathematics eds. M. Lachowicz and J. Miękisz

Vol. 80 Inelasticity of Materials: An Engineering Approach and a Practical Guide by A. R. Srinivasa and S. M. Srinivasan

Vol. 81 Stability Criteria for Fluid Flows by A. Georgescu and L. Palese

Vol. 82 Applied and Industrial Mathematics in Italy III eds. E. De Bernardis, R. Spigler and V. Valente

*To view the complete list of the published volumes in the series, please visit: http://www.worldscibooks.com/series/samas_series.shtml 
Series on Advances in Mathematics for Applied Sciences - Vol. 81

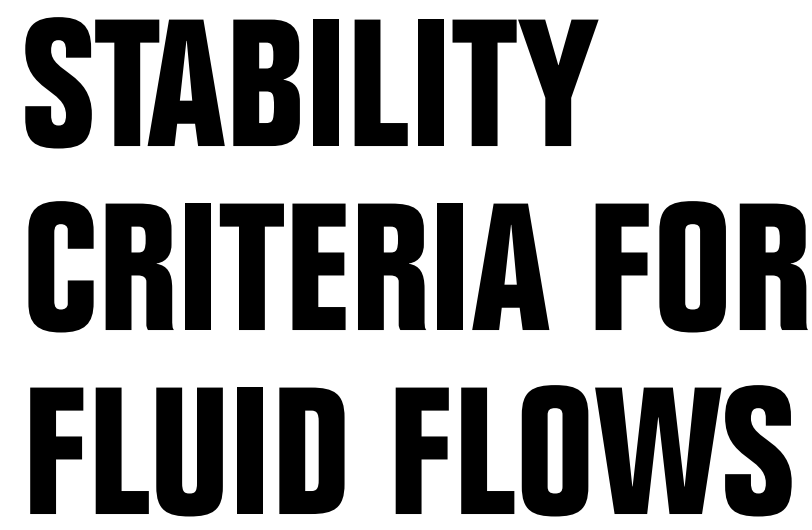

\section{Adelina Georgescu}

Academy of Romanian Scientists, Romania

Lidia Palese

University of Bari, Italy 
Published by

World Scientific Publishing Co. Pte. Ltd.

5 Toh Tuck Link, Singapore 596224

USA office: 27 Warren Street, Suite 401-402, Hackensack, NJ 07601

UK office: 57 Shelton Street, Covent Garden, London WC2H 9HE

\author{
Library of Congress Cataloging-in-Publication Data \\ Georgescu, Adelina. \\ Stability criteria for fluid flows / by Adelina Georgescu, Lidia Palese. \\ p. cm. -- (Series on advances in mathematics for applied sciences ; v. 81) \\ Includes bibliographical references. \\ ISBN-13: 978-981-4289-56-6 (hardcover : alk. paper) \\ ISBN-10: 981-4289-56-6 (hardcover : alk. paper) \\ 1. Heat--Convection--Mathematics. 2. Fluid mechanics--Mathematics. I. Palese, Lidia. II. Title.
}

QC327.G46 2009

536 '.25--dc22

2009026822

\title{
British Library Cataloguing-in-Publication Data
}

A catalogue record for this book is available from the British Library.

Copyright (C) 2010 by World Scientific Publishing Co. Pte. Ltd.

All rights reserved. This book, or parts thereof, may not be reproduced in any form or by any means, electronic or mechanical, including photocopying, recording or any information storage and retrieval system now known or to be invented, without written permission from the Publisher.

For photocopying of material in this volume, please pay a copying fee through the Copyright Clearance Center, Inc., 222 Rosewood Drive, Danvers, MA 01923, USA. In this case permission to photocopy is not required from the publisher. 


\section{Contents}

Introduction $\quad$ xi

1. Mathematical models governing fluid flows stability 1

1.1 General mathematical models of thermodynamics . . . . . . . . . . 1

1.1.1 Physical quantities and their mathematical description . . 1

1.1.2 Global quantities and their integral representation . . . . . 4

1.1.3 Balance equations in integral form . . . . . . . . . . . 5

1.1.4 Balance equations in differential form . . . . . . . . . 8

1.1.5 Constitutive equations. State equations . . . . . . . . . 9

1.2 Classical mathematical models in thermodynamics of fluids . . . . 15

1.2.1 Incompressible Navier-Stokes model . . . . . . . . . . . . . 15

1.2.2 Navier-Stokes-Fourier model and Oberbeck-Boussinesq approximation .................. 16

1.3 Classical mathematical models in thermodynamics . . . . . . . . . 18

1.4 Classical perturbation models . . . . . . . . . . . . . 20

1.4.1 Perturbation models . . . . . . . . . . . . . . . 20

1.4.2 Perturbation incompressible Navier-Stokes model . . . . . 20

1.4.3 Perturbation model for viscous incompressible homogeneous thermoelectrically conducting or nonconducting fluid 21

1.4.4 Perturbation model for viscous incompressible homogeneous thermoelectrically fully ionized conducting fluids . . 24

1.4.5 Perturbation model for viscous incompressible homogeneous thermoelectrically partially ionized conducting fluid 24

1.4.6 Perturbation model for a thermally conducting binary mixture in the presence of the Soret and Dufour effects . . . . 25

1.5 Generalized incompressible Navier-Stokes model . . . . . . . . . . 26

1.5.1 Generalized models . . . . . . . . . . . . . 26

1.5.2 Generalized model for strong solutions . . . . . . . . . 28

1.5.3 Perturbation generalized model for strong solutions . . . . 29 
2. Incompressible Navier-Stokes fluid 31

2.1 Back to integral setting; involvement of dynamics and bifurcation . 31

2.2 Stability in semidynamical systems . . . . . . . . . . . . . 33

2.3 Perturbations; asymptotic stability; linear stability . . . . . . . . . 39

2.4 Linear stability . . . . . . . . . . . . . . . . . . . . . . . . . . . . . . . . 40

2.4.1 Finite-dimensional case . . . . . . . . . . . . . . 41

2.4.2 Infinite-dimensional case . . . . . . . . . . . . . . . . . . . . . . . . . . 57

2.5 Prodi's linearization principle . . . . . . . . . . . . . . 50

2.6 Estimates for the spectrum of $\tilde{A} \ldots \ldots \ldots$. . . . . . . . . . . . 54

2.6.1 Necessary conditions for belonging to $\sigma(-\tilde{A}) \ldots . . . . .55$

2.6.2 Spectrum bounds based on straight lines . . . . . . . . . 57

2.6.3 Spectrum bounds based on parabolas . . . . . . . . . . 60

2.7 Universal stability criteria . . . . . . . . . . . . . . . . 62

2.7.1 Energy relation . . . . . . . . . . . . . 62

2.7.2 Three-dimensional case . . . . . . . . . . . 66

2.7.3 Two-dimensional case . . . . . . . . . . . . . . 69

3. Elements of calculus of variations $\quad 75$

3.1 Generalities ...................... 75

3.2 Direct and inverse problems of calculus of variations . . . . . . . 80

3.2.1 Variational problems in classical, generalized and abstract setting .................... 80

3.2.2 Construction of the boundary-value problem associated with a variational problem. Necessary conditions for extremum .................. 83

3.2.3 Classical Euler equations associated with variational problems for particular functionals . . . . . . . . . . 85

3.2.4 Construction of the variational problem associated with an Euler equation: energy method. Quadratic functionals associated with affine or linear equations . . . . . . . . . . . 92

3.2.5 Direct methods. Minimizing sequences . . . . . . . . . 102

3.2.6 General isoperimetric problems and inequalities and associate eigenvalue problems . . . . . . . . . . . 106

3.2.7 Energy method for non-stationary equations . . . . . . . . 111

3.3 Symmetrization of some matricial ordinary differential operators . 117

3.3.1 Four eigenvalue problems of thermal convection . . . . . . 118

3.3.2 Adjoint operators, their symmetric and skew-symmetric part for matricial ordinary differential operators defining problems (3.3.1)-(3.3.7) . . . . . . . . . . . . 121

3.3.3 Symmetrization of matricial ordinary differential operators defining problems $(3.3 .1)-(3.3 .7)$. . . . . . . . . . . 124

3.4 Variational principles for problems (3.3.1)-(3.3.7) . . . . . . . . 129 
3.4.1 Boundary-value problems versus associated variational functional . . . . . . . . . . . . . 129

3.4.2 Variational principles for the first three two-point problems for $(3.3 .1)-(3.3 .3) \ldots \ldots \ldots \ldots$. . . . . . . . . . 131

3.4.3 Variational principles for problem (3.3.1)-(3.3.3), (3.3.7) . 133

3.5 Fourier series solutions for variational problems . . . . . . . . . . 145

4. Variants of the energy method for non-stationary equations 151

4.1 Variant based on differentiation of parameters . . . . . . . . . . . . 151

4.1.1 Classical mathematical model governing the conduction and convection in a binary mixture . . . . . . . . . . . 151

4.1.2 Perturbation model . . . . . . . . . . . . . . . 153

4.1.3 Energy relation . . . . . . . . . . . . . . . . 155

4.1.4 Energy inequality and the stability criterion . . . . . . . 158

4.1.5 Case $N=\lambda=0, s=1 \ldots \ldots$. . . . . . . . 160

4.1.6 Case $N=\lambda=0, s=-1 \ldots \ldots$. . . . . . . 161

4.1.7 Coincidence of nonlinear (energy) and linear stability limits 162

4.2 Variant based on simplest symmetric part of operators . . . . . . . 168

4.2.1 Symmetry and optimality condition . . . . . . . . . . . 168

4.2.2 More general setting of our variant . . . . . . . . . . . 171

4.2.3 Symmetry instead of optimality condition (4.2.23) . . . . . 174

4.2.4 Case of nonsymmetric operators in a horizontal convection problem . . . . . . . . . . . . . . . . 177

4.2.5 Case of new vector unknown functions in a vertical hydromagnetic convection . . . . . . . . . . . . . 180

4.3 Variants based on energy splitting . . . . . . . . . . . . . . 183

4.3.1 Splitting of Lyapunov functional . . . . . . . . . . . . . . . 183

4.3.2 Nonlinear stability of the MHD anisotropic Bénard problem 186

5. Applications to linear Bénard convections 199

5.1 Magnetic Bénard convection in a partially ionized fluid . . . . . . . 200

5.1.1 Mathematical results for the magnetic Bénard problem . . 201

5.1.2 Splitting of the two-point eigenvalue problem . . . . . . . . 202

5.1.3 Neutral curves for the even case . . . . . . . . . . . . . . . 204

5.1.4 Neutral curves for the odd case . . . . . . . . . . . . 209

5.1.5 Thermosolutal instability of a compressible Soret-Dufour mixture with Hall and ion-slip currents in a porous medium 212

5.2 Magnetic Bénard convection for a fully ionized fluid . . . . . . . . 222

5.2.1 Neutral curves for the even case . . . . . . . . . . . . . 222

5.2.2 Neutral curves for the odd case . . . . . . . . . . . . . 226

5.3 Convection in a micro-polar fluid bounded by rigid walls . . . . . . 231

5.4 Convections governed by ode's with variable coefficients . . . . . . 233 
5.4.1 Deep convection ................... . 234

5.4.2 Convection in a variable gravity field . . . . . . . . . . 237

5.4 .3 Penetrative convection . . . . . . . . . . . . . . 241

5.4 .4 Convection with a heat source in a rigid box . . . . . . . 243

6. Variational methods applied to linear stability 247

6.1 Magnetic Bénard problem with Hall effect . . . . . . . . . . . . . . 248

6.1.1 Reformulation of the evolution equations of perturbations as an integro-differential equation . . . . . . . . . . . 249

6.1.2 The associated functional and Euler equations . . . . . . . 251

6.1.3 Stability criteria . . . . . . . . . . . . . . 253

6.1.4 Concluding remarks and a list of formulae used in Section $6.1 .3 \ldots \ldots \ldots \ldots \ldots . \ldots \ldots 256 \ldots$

6.2 Lyapunov method applied to the anisotropic Bénard problem . . . 258

6.2.1 Energy relation for the Lyapunov (energy) functional . . . 258

6.2.2 Associated Euler-Lagrange equations and the secular equation . . . . . . . . . . . . . . 260

6.2.3 Positive definiteness of the Lyapunov functional $E_{l} \quad \ldots$. . 261

6.2.4 Stability criteria . . . . . . . . . . . . . . . 261

6.3 Stability criteria for a quasi-geostrophic forced zonal flow . . . . . 263

6.3.1 Perturbation model . . . . . . . . . . . . . . 263

6.3.2 Energy inequality . . . . . . . . . . . . . . . . . 264

6.3.3 Criteria expressed in terms of the maximum basic vorticity $\mu_{2}$ only. Use of $(6.3 .11) \ldots \ldots \ldots$. . . . . . . . 266

6.3.4 Criteria in terms of $\mu_{2}$ only. Use of (6.3.12) . . . . . . . 267

6.3.5 Criteria in terms of $\mu_{2}$ and $\mu_{3} \ldots \ldots$. . . . . . 268

6.3.6 Criteria in terms of $\mu_{2}$ only. Use of (6.3.11) and (6.3.12) . 269

6.4 Variational principle for problem (5.3.1), (5.3.2) . . . . . . . . . 269

6.4.1 Variational principle for the case $Q=\bar{\delta}=0, A, R \neq 0$. . . 270

6.5 Taylor-Dean problem . . . . . . . . . . . . . . . 271

6.5.1 Eigenvalue problem . . . . . . . . . . . . . . 271

6.5.2 Reformulation of (6.5.2), (6.5.3) as a selfadjoint problem and the associated variational principle . . . . . . . . 273

6.5.3 Variational principle for the non-selfadjoint problem

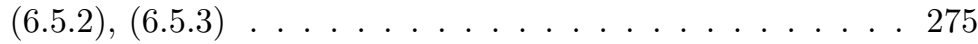

7. Applications of the direct method to linear stability 277

7.1 Couette flow between two cylinders subject to a magnetic field . . 277

7.1.1 Characteristic equation and its bifurcation sets . . . . . . . 278

7.1.2 Secular equations dependent on boundary conditions . . . 280

7.1.3 Secular equations and points; independence of boundary conditions . . . . . . . . . . . . . . . 281 
7.1.4 Open problems for (7.1.1) and (7.1.2) . . . . . . . . 283

7.2 Soret-Dufour driven convection . . . . . . . . . . . . . . 283

7.2.1 Equations satisfied by the unknown functions . . . . . . . 283

7.2.2 Case $a=0$ and case $k=0$. . . . . . . . . . . . 286

7.2 .3 Case $k=-a^{4} \ldots \ldots$. . . . . . . . . . . . 287

7.3 Magnetic Soret-Dufour driven convection . . . . . . . . . . . 288

7.3.1 Eigenvalue problem . . . . . . . . . . . . . 288

7.3.2 Case $M>0$. Double roots of the characteristic equation . 289

7.3.3 Case $M=0$. . . . . . . . . . . . . . 291

7.4 Convection in a porous medium . . . . . . . . . . . . 292

7.4.1 Lower rigid isothermal surface, upper free isolated surface 292

7.4.2 Two free isothermal surfaces . . . . . . . . . . . . . . 298

7.5 Convection in the presence of a dielectrophoretic force . . . . . . . 300

7.6 Convection in an anisotropic M.H.D. thermodiffusive mixture . . . 301

7.6.1 Formulation of the eigenvalue problem . . . . . . . . 301

7.6.2 Case $a=0$ of the perturbations depending only on the vertical coordinate $z$ and time . . . . . . . . . . 303

7.6.3 Case $a \neq 0, M=0, b_{1}=0 \ldots 304$

7.6.4 Case $a \neq 0, M^{*}=0, R^{*} \neq 0 \ldots 305$

7.6.5 Case $a \neq 0, M^{*}>0, R^{*}=0 \ldots 306$

7.6.6 General case $a \neq 0, M^{*}>0, R^{*} \neq 0 \ldots 309$

7.6.7 Open problems................ 311

7.7 Inhibition of the thermal convection by a magnetic field . . . . . . 311

7.7.1 Multiplicity of the characteristic roots . . . . . . . . . 312

7.7 .2 Secular equations . . . . . . . . . . . . . 313

7.8 Microconvection in a binary layer subject to a strong Soret effect . 315

7.8.1 Eigenvalue problem . . . . . . . . . . . . . 315

7.8.2 Characteristic equation and its bifurcation set . . . . . . 316

7.8.3 False secular points . . . . . . . . . . . . . . 319

7.9 Convection in the layer between the sea bed and the permafrost . 319

$\begin{array}{lll}\text { Appendix } 1 & \text { Sets with structure } & 323\end{array}$

$\begin{array}{lll}\text { Appendix } 2 & \text { Operators and functionals } & 337\end{array}$

Appendix 3 Differential operators in $L^{2}(a, b)$

Appendix 4 Differential operators in $\left(L^{2}(a, b)\right)^{n} \quad 353$

$\begin{array}{lll}\text { Appendix } 5 & \text { Fourier series expansions } & 361\end{array}$

Appendix 6 The direct method based on the characteristic equation $\quad 369$ 
Appendix 7 First and second order differential matricial operators 


\section{Introduction}

In this volume, the main methods, techniques and tricks used to derive sufficient conditions for fluid flow stability are discussed. In general, nonlinear and linear cases require different treatments, thus we have to differentiate between linear and nonlinear criteria.

With a few exceptions, the treatment is analytical, but connections with the geometric viewpoint of dynamical systems are also outlined.

Inequalities and their use are crucial for finding stability criteria. That is why particular attention is paid to classical or generalized analytical inequalities, especially to those relating integrals of functions and their derivatives. The best constants involved into the last ones can be viewed as extrema of some associated functionals. If the extrema are with constraints, the corresponding inequalities are the so-called isoperimetric inequalities. Further, in order to solve the associated variational problems, direct methods, based on expansions in Fourier series upon total sets of functions, turned out to be among the most efficient. The Fourier series can be introduced directly into the functional or into the corresponding Euler equations, which, in the isoperimetric case, are eigenvalue problems. Moreover, the expansion functions may be chosen to satisfy all boundary conditions of the problem or part of them (especially when even and odd derivatives occur in equations or/and boundary conditions). Finally, in looking for variational principles natural conditions may occur. Several variational aspects related to functional inequalities used to prove stability criteria emerge, to justify the insertion of an entire chapter (3) devoted to variational problems. Algebraic and differential inequalities are summarized in Appendix 1 together with some formulae of tensor analysis.

A great amount of hydrodynamic and hydromagnetic stability criteria exist, and we do not intend to present them all, having chosen to limit ourselves to the founder's criteria, our own results, and a few other results of the Italian and Romanian schools in the field, for mixtures and in the Bénard magnetic case, for free or rigid walls. In addition, we are concerned mainly with convection problems 
(including temperature; concentration; magnetic, Soret, Dufour, Hall, ion-slip, dielectrophoretic effects in horizontal layers) for viscous fluids or fluid mixtures. Only in a few cases, horizontal convection and other effects are considered.

In most cases, we use variational methods, methods of Hilbert spaces theory, methods based on inequalities (isoperimetric or not), the Fourier series method and a direct method based on the characteristic equation. This is why, with a few exceptions, in the linear cases treated by us, the ordinary differential equations (ode's) have constant coefficients but a higher order. In the nonlinear case, for the sake of simplicity, in order to have a symmetrizable linearized part, we preferred basic equilibria or steady flows described or approximated by affine functions. Consequently, this book considers fluid flows whose stability properties do not depend on local phenomena.

Nowadays, hydrodynamic stability theory is involved in important ecological and industrial problems, requiring a lot of effects, and characteristics of fluids configurations, other than traditional ones, being taken into account. Among them we quote: rotation in spherical and cylindrical configurations; capillarity; heat sources; magneto-elastic effects; compressibility; chemical reactions; temperature-dependent viscosity, nonlinear (e.g. cubic) dependence of temperature on the density; porosity; variable gravity; liquid drops; fluids heated rapidly in a time-dependent manner; finite conductivity of the walls; surface films in Bénard convection; concentration and temperature-dependent surface tension; impulsive heating or cooling; heating from above; uniformly accelerated fluids; sound effects in convection; time-dependent basic flows; multi-component fluid layers; physically nonlinear fluids or other continua (second grade fluids, simple fluids, dissociating fluids); fluids with structure (dipolar fluids, micro-polar fluids, ferromagnetic fluids); multiphasic continua and phase transition in continua (mixed phases, melting, mushy zones, frozen zones, phase changing snow packs, ice streets, glaciers directional solidification, solid friction); dynamo. We treat a few of these complex problems in a didactic way, in order to be useful to other similar topics. We do not repeat classical and, by now, simple results of hydrodynamic and hydromagnetic stability theory. They can be found in the basic monographs on the topic: [Lin], [Chan], [DraR], [J76], [Geo85], [Yu2], [Ioo2], [Kos]. We go further instead with more complex but still basic subjects, e.g. linearization principle, universal stability criteria, stability spectrum estimates, variational principles, improved energy methods, treatment of problems with intricate boundary conditions.

The treatment in Chapters 1-3 differs from that in Chapters 4-7. Each section in Chapters 4-7 concerns a single physical case and the presented cases are quite complicated. Most of them include several physical effects (e.g. mechanical, thermal, magnetic, chemical, porosity) and several mathematical methods (e.g. differential and/or integral inequalities, tensorial formulae, variational problems, eigenvalue problems). For each case the physical motivation and conditions are provided. On the contrary, in Chapter 1, a more modern physical basis of mathe- 
matical modelling of fluid motions is given. Chapter 2 yields basic mathematical results on the stability involved in the resulted models. Chapter 3 is a brief presentation of needed variational arguments through simple examples whose physical bases are briefly explained. The proofs of the variational results are only sketched. In exchange, comments on their validity are carefully presented.

Moreover, for all worked examples, detailed computations are given.

Throughout the book we try as much as possible to treat the most realistic cases. Firstly, this is related especially to the boundary conditions which "spoil the symmetry" of the mathematical problem, the given problem being reformulated so that elegant functional analytic methods apply to the new setting. Secondly, we bring into actuality some other powerful approaches (e.g. Budiansky-DiPrima (BD) method, backward integration method) frequently used several decades ago, and almost forgotten by now by applied mathematicians. Thirdly, we call the attention of mathematicians interested in fluid flow stability towards powerful methods having an engineering flavor (e.g. Joseph's differentiation of parameters approach, B-D method, the direct method).

Minimal prerequisites are: fundamentals of classical calculus of variations (Euler equations, isoperimetric problems, variational principles), linear and nonlinear functional analysis (normed spaces, symmetric operators, generalized derivatives, embedding theorems in Sobolev spaces, quadratic functionals, energy spaces), tensor analysis (flux-divergence formula, representation formulae for solenoidal and poloidal vectors, calculus with differential operators). Their brief presentation can be found in appendices and in Section 3.2.

The book is mainly addressed to applied mathematicians and other researchers in hydrodynamic and hydromagnetic stability. It can also be a companion paper to courses in these fields, e.g. [Geo85].

Chapter 1 presents briefly the modelling and the linear and general mathematical models of thermodynamics of fluids in the I. Müller framework. Then the particular classical models used in the book and their corresponding perturbation models around a steady solution are written. The generalized settings of some classical models are provided too.

Chapter 2 deals with geometric reframing of analytical models of hydrodynamic stability theory in dynamical system theory as evolution equations in Banach spaces in view of their use in the linearization principle and energy method in the nonstationary case. Physical and mathematical concepts of energy leading to different definitions of stability are discussed. Improvements of the Prodi estimations for the spectrum of the operator defining the linearized incompressible Navier-Stokes (N-S) equations are obtained, enabling us to derive some universal stability criteria. Some others are obtained by a better use of integral inequalities in a generalized form of the N-S equations. Bounds for linear stability which are also criteria for nonlinear stability are given. Below them, no perturbation amplifies. If these bounds become limits of linear stability, then the existence of subcritical instabilities is excluded. 
This is quite a rarely treated case. We considered it in Chapter 4 .

Chapter 3, the core of the book, is a systematic presentation of the results of the calculus of variations used in hydrodynamic and hydromagnetic stability theory. First it introduces the two main traditional topics in calculus of variations, i.e. variational problems and Euler equations, then shows their relationship revealed by means of the energy method, cases where they are equivalent, and their numerical realization. Due to their notable involvement in deriving stability criteria, the isoperimetric problems, associated isoperimetric inequalities and eigenvalue problems are separately dealt with. Then all these variational methods are exemplified by four two-point problems for a system of three ode's governing the linear stability of some mechanical equilibrium of a fluid: The nonsymmetric matricial differential operators are associated, then they are symmetrized, variational principles for the obtained equations are written, and the use of Fourier series expansions yields stability criteria. This first part provides powerful tools for the investigation of stationary problems. A distinct topic, namely energy method for evolution equations, suited to non-stationary problems, is then dealt with. A bridge between the two tools is represented by isoperimetric inequalities, deduced by stationary tools but used in the non-stationary case.

Chapter 4 deals only with three variants of the non-stationary energy method and their application to concrete nonlinear convection problems governed by pde's. We present only those variants closely related to our own. The first variant is Joseph's method and it was developed by him for a thermal convection problem in the case of a binary fluid mixture, characterized by one vector (velocity) and two scalar (temperature and concentration) unknown functions. His first basic idea was to define a new energy capturing the contribution of some variable-sign terms from the energy relation. This was achieved by introducing as new scalar unknown functions some linear combinations of temperature and concentration. The second idea was to establish a relationship between the undetermined coefficients (parameters) such that the energy relation contains no derivatives of any new scalar functions. The third main idea was to derive the constraint on the parameters in order for the stability bound to be optimal. In Section 4.1 we present our extension of Joseph's method for a convection problem containing an additional interaction between thermal diffusivity and diffusive thermal conductivity. Joseph's lines are followed, but instead of using as unknown parameters the coefficients in the above-mentioned combinations, we use the coefficients in the inverse combinations, yielding a much easier computation. In Section 4.2 the Georgescu-Palese-Redaelli (G-P-R) method is described. It generalizes Joseph's approach translating his ideas in terms of symmetrization of the operators occurring in some equations equivalent to the governing equations and derived from them by suitable scalar multiplications. It is found that the linear and nonlinear stability limits coincide, proving the equivalence of Joseph's and our method. Even more efficient approaches are given in Sections 4.2.2 and 4.2.3. In them we use only the optimality Joseph condition, all other arguments 
being new. In Section 4.2.4 a horizontal convection is analyzed. For a vertical hydromagnetic convection problem in Section 4.2.5 the G-P-R method is extended to the case of two vector and one scalar unknown functions. The variants based on the energy splitting are sketched in Section 4.3.1. Some of the ideas developed in Section 4.3.1 are applied in Section 4.3.2 to a very complicated hydromagnetic convection problem, whose solution uses our ideas from the linear case (Section 6.2).

Chapters 5 and 6 provide linear stability results mainly obtained by our group using the direct (Chapter 5) or variational (Chapter 6) B-D method. The perturbation fields are taken in the form of normal modes, reducing the problem to a one-dimensional case. Further the B-D method applies: the unknown functions are expanded in Fourier series on sets of sines and cosines which are total in some separable Hilbert subspaces of some Sobolev spaces. In fact, we tried to keep as close to the theory of these spaces as possible in view of avoiding any earlier approximation and its uncontrollable error propagation due to the cumbersome computations involved. In this way, the boundary value problem for a system of ode's becomes an algebraic system in the Fourier coefficients.

Unlike other methods based on Fourier series, in the B-D method the expansion functions are easy to construct and satisfy only part of the boundary conditions of the problem. The other boundary conditions introduce some constraints, to be satisfied by the Fourier coefficients, leading to secular equation defined by a finitedimensional determinant each entry of which is a converging series. This equation is exact and easier to compute in comparison with the standard secular equation defined by a determinant of infinite order and corresponding to ChandrasekharGalerkin methods, where the expansion functions satisfy all boundary conditions. Supplementary simplifications are obtained by splitting the problem into even and odd problems. These advantages show that the B-D method is appropriate to flows subject to several physical effects and to complicated boundary conditions, when the standard methods are practically nonapplicable. In Chapter 5 we illustrate them on complicated cases of convections, involving many unknown functions and parameters, various boundary conditions and high order derivatives which can be of even and odd order. There the B-D method is applied directly to the equations.

In Chapter 6, a variational principle is first shown to hold, and then the series expansions are introduced into the functional. As this functional involves a smaller number of derivatives, the computations are easier the closer is the operator defining the equations to a symmetric operator. Some results obtained by means of the B-D method are the best and, sometimes, the only ones to be found in literature, pleading for the reconsideration of this method. It was applied to stability of elastica in the '40-s and in the '60-s to fluid flow stability. Subsequently, it was almost forgotten until its systematic use by our group.

The transition from the generalized treatment of nonlinear pde's (Chapters 2-4) to the (apparently) classical study of linear ode's (Chapters 5-7) is somehow abrupt. However, the linearized equations are often involved and used in the first step of 
nonlinear approaches and in their numerical realization based on the eigenvalues of linear problems.

Moreover, in the absence of a quite general linearization principle, comparing nonlinear and linear stability limits is necessary to the physical interpretation of the results. Chapter 5 realizes a "translation" of properties of ode's into those of the associated algebraic system of Fourier coefficients of their solutions. Further, Chapter 6 provides equivalent variational settings for eigenvalue problems defined by matricial differential operators. The direct method applied to several fluid flows in Chapter 7 yields complementary results to linear problems dealt with by methods from Chapters 5 and 6 . Among them there are two topics rarely treated in the literature, namely the detection of false neutral manifolds and of the secular equations independent of the boundary conditions. The involved investigation of the multiplicity of the characteristic roots allows one the determination of the true secular equations and, consequently, the detection of false neutral manifolds (occurring in formal direct use of numerical methods). Various approaches to study this multiplicity are shown and the associated bifurcation sets are revealed. The complex cases treated in Chapter 7 by the direct method show its adequacy to real-world problems involving any type of boundary conditions, many parameters and high orders of differentiation. Providing closed-form solutions as a sum of a small number of terms, this method enables one to find secular equations independent of the boundary conditions. In this way, some paradoxical behaviors of certain eigenvalue perturbation problems are explained.

In this book the formulae, theorems, remarks, definitions, lemmas are labeled by three numbers, while in the appendices, by two numbers. The physical quantities occurring in the book are listed at the end of Section 1.1.1.

Apart from section titles, the following abbreviations are frequently used in the text: Budianski-DiPrima (B-D), Navier-Stokes (N-S), Navier-Stokes-Fourier (N-S$\mathrm{F}$ ), Oberbeck-Boussinesq (O-B), ordinary differential equation (ode), partial differential equation (pde).

We are deeply indebted to Mihnea Moroianu for reading the entire manuscript and making valuable improvements. We also kindly acknowledge the remarks and suggestions made by our collaborators Catalin-Liviu Bichir, Ioana-Florica Dragomirescu, Arcangelo Labianca and Aldo Redaelli.

The authors 